\title{
Second Harmonic Generation in the Background of Photon Counting
}

\author{
KURILLA Boldizsár ${ }^{1}$
}

\begin{abstract}
This article will show an application of photon counting using an experiment with a Second Harmonic Generation (SHG) to take a look at the basics of single photon communication. This will be an opportunity to open the way for long distance single photon laser communication between unmanned robotic vehicles. This article also gives an insight into a similar, but smaller setup towards the application on unmanned robotic vehicles. In this work measurements by SHG from Si (111) sample have been done with all polarization configurations with the help of a MIRA 900 type Ti: Sapphire laser. The other part of the experiment was carried out with an SR 400 photon counter device which was used for counting the impacting pulses with the help of a photomultiplier tube (PMT).
\end{abstract}

Keywords: Second Harmonic Generation, photon counting, laser communication, photomultiplier tube

\section{Introduction}

Photon counting is a process for laser communication applications in the field of laser physics. Photon counting is becoming a very important process in long distance quantum communication experiences. It was already demonstrated between two islands of the Canary Islands that quantum communication with single photons over $100 \mathrm{~km}$ is possible. [1]

Future laser communication needs smaller laser devices to achieve communication with quantum secrecy on board unmanned robotic vehicles. Most of today's laser physical devices are too large and heavy to install on small unmanned robotic vehicles; in addition to needing high energy to operate.

Second harmonic generation (SHG; also called frequency doubling) is a nonlinear optical process, in which photons interacting with a nonlinear material are effectively "combined" to form new photons with twice the energy, and therefore twice the frequency and half the wavelength of the initial photons. SHG contains a very special field in nonlinear optics and it is also a special case of sum frequency generation.

\section{Basics of Second Harmonic Generation}

To understand the basics of SHG, the conduction must be based on the fact, that the polarization is aligned with and proportional to the E electric field in an isotropic dielectric, linear and homogeneous medium that can be expressed by the following equation:

1 kurilla.boldizsar@gmail.com 


$$
P=\varepsilon_{0} \chi E
$$

where $\varepsilon_{0}$ is the electric constant, and $\chi$ is the electric susceptibility of the medium. [2]

In an anisotropic material, the polarization and the field are not necessarily in the same direction. Then, the $i^{\text {th }}$ component of the polarization is related to the $j^{\text {th }}$ component of the electric field according to:

$$
P_{i}=\sum_{j} \varepsilon_{0} X_{i j} E_{j}
$$

where $\varepsilon_{0}$ is the electric constant and $\chi_{i j}$ is the electric susceptibility tensor of the medium.

This relation shows, that a material can polarize in direction $\mathrm{x}$ by applying a field in direction $\mathrm{z}$, and so on.

In general the power series of applied electric field $\mathrm{E}$ should be expressed by the polarization of the medium. As it has been already realized, the electric polarization induced in the medium. [3]

$$
P=\varepsilon_{0}\left[\chi^{(1)} \cdot E(\omega)+\chi^{(2)}: E(\omega) E(\omega)+\chi^{(3)}: E(\omega) E(\omega) E(\omega)+\ldots\right]
$$

where $\mathrm{P}$ is the nonlinear electric polarization in the material, $\varepsilon_{0}$ is the electric permittivity of free space $(\mathrm{F} / \mathrm{m})$ and $\chi^{(i)}$ is the $i$ th order susceptibility tensor of the medium. [3]

\section{Types of SHG}

Second harmonic generation occurs in two types, denoted I and II. In Type I SHG two photons having ordinary polarization with respect to the crystal will combine to form one photon with double the frequency and extraordinary polarization. In Type II SHG, two photons having orthogonal polarizations will combine to form one photon with double the frequency and extraordinary polarization. For a given crystal orientation, only one of these types of SHG occurs.

Investigation of the SHG principles (including the PMTs and photon counting systems) has been done to understand the phenomenon itself. SHG is a phenomenon where an input wave in a nonlinear material can generate a wave with twice the optical frequency. [2] In special cases the SHG phenomena will allow a very high axial and lateral resolution that can give productive signals from tunable nanostructures. [4] There is also an aim to study the polarization dependence in SHG, because it provides information on the orientation of molecules composing or absorbed on the surface.

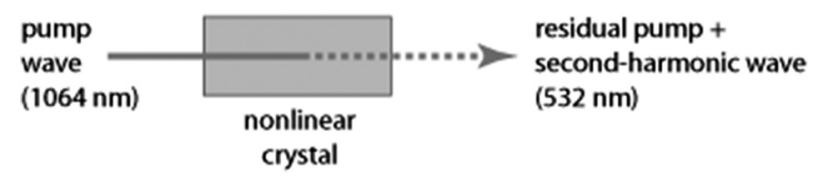

Figure 1. Typical configuration for frequency doubling: an infrared input beam at $1064 \mathrm{~nm}$ generates a green $532 \mathrm{~nm}$ wave during its path through a nonlinear crystal. [5] 
SHG has a great importance in the field of metal nanoparticles. SHG is a surface sensitive optical probe where the metallic nanoparticles (like gold) are very good substrates for measurements because generally metallic nanoparticles have very large optical resonances. [6]

For centrosymmetric systems SHG is forbidden, because of symmetry considerations and thus strongly depend on small deviations from the symmetric shape as well as from broken symmetry at interfaces. It is important to know that metallic nanoparticles show localized surface plasmon resonances (LSPRs). It leads to strong absorption/scattering and local field enhancement near these kinds of structures. [7] These nanoparticles have linear optical properties that have been dominated by collective oscillations of the conduction electrons. It has already been demonstrated on gold nanorods by spectral studies that SHG strongly depends on the incident light polarization direction. Its excitation spectroscopy clearly proves the role of LSPR in the SH signal enhancement.

\section{The setup of SHG}

In Fig. 2 the optical second harmonic generation setup and the MIRA 900 Ti:Sapphire laser are aligned. The MIRA $900 \mathrm{Ti}$ :Sapphire laser is a pulsed laser with $82 \mathrm{MHz}$ frequency and has $100 \mathrm{fs}$ repetition rate. The laser is sensitive to temperature changes so it must be realigned many times. To find the strongest and clearest signals, it was obligatory to find the best focus on the sample and it needed to avoid any unwanted light interaction. The power of the laser must be also optimized. The photomultiplier tube (PMT) has been connected to the preamplifier and finally to the SR400 photon counting system.

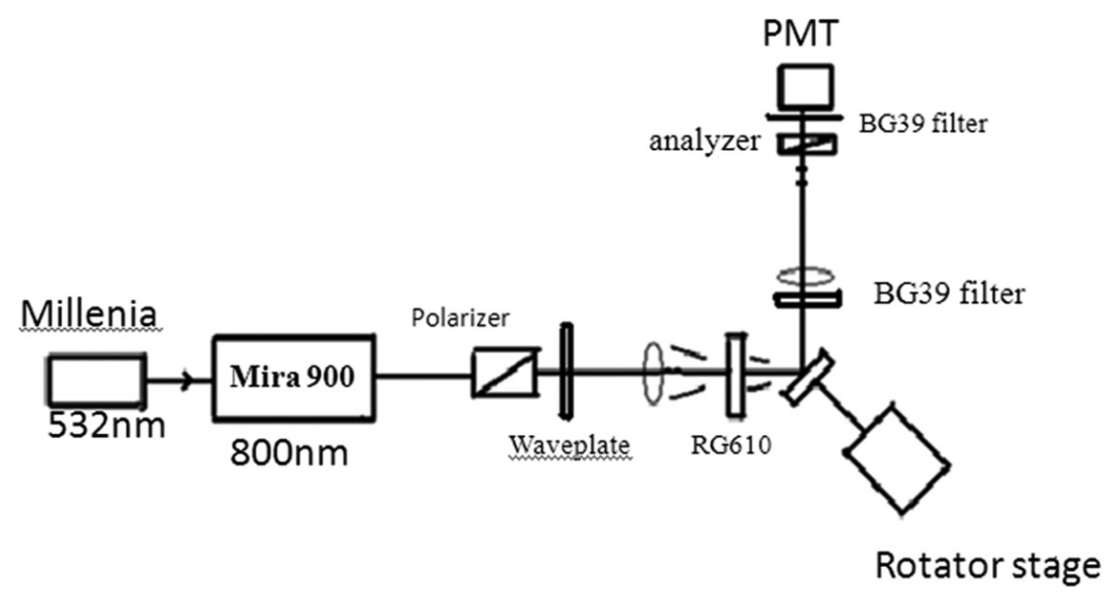

Figure 2. Sketch of the optical second harmonic generation setup. (Made by author.)

A PMT is useful for light detection of very weak signals. It is a photoemissive device in which the absorption of a photon results in the emission of an electron. These detectors work by amplifying the electrons generated by a photocathode exposed to a photon flux. The laser polarization is checked with the Brewster angle (horizontal). The Mira 900 Ti:sa laser (800 nm) is pumped by the Millenia Pro laser (532 nm). The driving engine is a Neodymium Yttrium Vanadate (Nd:YVO4) laser crystal. It is capable of producing 5.5 Watts of $1064 \mathrm{~nm}$ 
laser beam. Frequency-doubling converts the $1064 \mathrm{~nm}$ light to a green $532 \mathrm{~nm}$ light that becomes the output of the laser. The Millenia is pumped by two diode lasers located in the power supply. The system contains a polarizer and an analyser for checking the signals at all polarization configurations. If the vibrations of the polarized light are parallel to the plane of incidence, the light is said to be p polarized and if the vibrations of the polarized light are perpendicular to the plane of incidence, the light is said to be s polarized. Next to the sample we also have to use some special short wave pass filters to avoid any other noises. The setup contains a half wave plate too, which retards one polarization by half a wavelength, or 180 degrees. This changes the polarization direction of linear polarized light. The sample reflects the incident light by 45 degrees. After it passes through another lens, that makes the beam parallel, it passes through the BG39 type blue filters to let only the $400 \mathrm{~nm}$ wavelength light be detected by the PMT. In Picture 1, the SHG setup can be seen just like in the sketch above. The rotator stage holds the sample and it can be used to rotate the sample holder to make the measurements by 1 degrees step size.

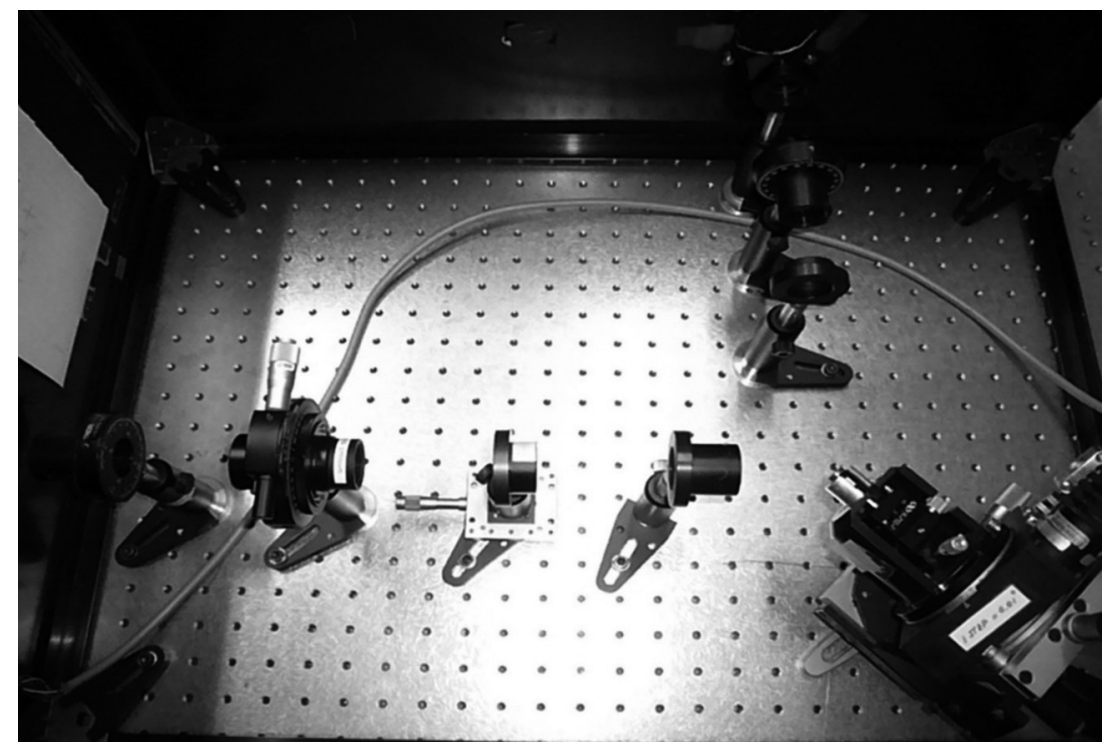

Picture 1. The second harmonic generation setup. (Made by author.)

\section{Principles of photon counting}

Inside the PMT photoelectrons emitted from the photocathode are accelerated and focused onto the first dynode to produce secondary electrons. However, some of these electrons do not strike the first dynode or deviate from their normal trajectories, so they are not multiplied properly. This efficiency of collecting photoelectrons is referred to as the collection efficiency (CE). The ratio of the count value (the number of output pulses) to the number of incident photons is called the detection efficiency (DE) or counting efficiency, and is expressed by the following equation:

$$
\operatorname{DE}(\%)=\left(\frac{\mathrm{Nd}}{\mathrm{Np}}\right) \cdot 100 \%=\eta \cdot \alpha \cdot 100(\%)
$$


where $\mathrm{Nd}$ is the count value, $\mathrm{Np}$ is the number of incident photons; $\eta$ is the photocathode quantum efficiency (QE) and $\alpha$ is the CE. [8] Quantum efficiency is the production probability of photoelectrons being emitted when one photon strikes the photocathode. In our case the QE of our PMT is approximately $25 \%$ at $400 \mathrm{~nm}$ and the PMT gain (G) is $10^{7}$. Detection efficiency also depends on the threshold level that brings the output pulses into a binary signal.

The dark count (DC) of the PMT is the number of residual counts arising from radioactive decays of materials inside the PMT, and from cosmic rays. The primary noise source is thermionic emission of electrons from the photocathode. To dramatically reduce the dark count rate, the thermoelectric cooling of the PMT is extremely important. A standard PMT works with good efficiency at $-20^{\circ} \mathrm{C}$. At this temperature the dark count rate can be reduced from a few $\mathrm{kHz}$ to a few $\mathrm{Hz}$. The dark count rate also depends on the cathode type and it is highest for cathodes with high sensitivity at long wavelengths. [9][10]

PMTs will specify their noise in terms of the rate of output pulses. The noise is specified as an anode dark current ( $\mathrm{DCu}$ ). Assuming the primary source of dark current is thermionic emission from the photocathode, the dark count rate is given by the following expression:

$$
D C(k H z)=\frac{6 \cdot \mathrm{DCu}(\mathrm{nA})}{\mathrm{G}}
$$

where $\mathrm{G}$ is the gain of the PMT. [10]

Picture 2 shows the SHG setup connected to the PMT that is connected to an SR445 type preamplifier.

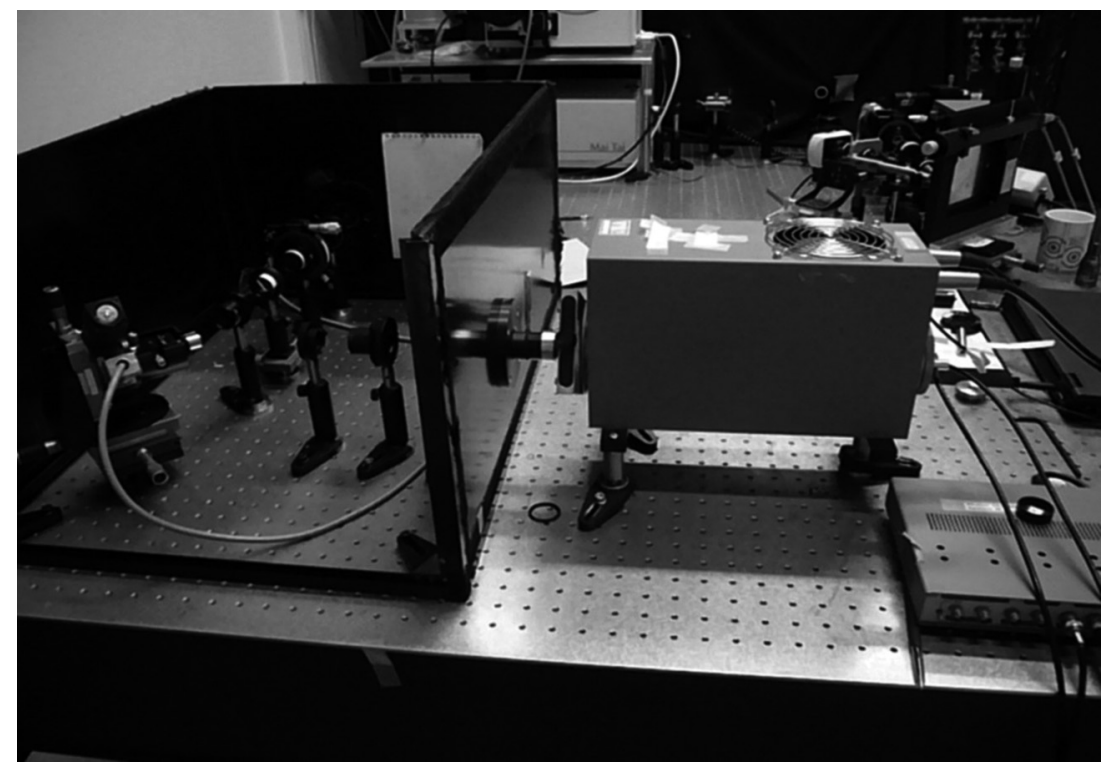

Picture 2. The second harmonic generation setup and the PMT. (Made by author.) 
From the first dynode the number of emitted secondary electrons is approximately 20 in response to one primary electron from the photocathode. In general they can be treated by Poisson distribution, and the average number of secondary electrons becomes the secondary electron emission ratio $\delta$. In the subsequent dynodes it holds true for multiplication processes. To create a group of electrons, a single photoelectron from the photocathode is multiplied by $\delta^{2}$ and is derived as an output pulse from the anode due to the fact that a PMT has n stages of dynodes. The height of each output pulse obtained at the anode depends on fluctuations in this process in the secondary electron multiplication ratio stated above, so that it differs from pulse to pulse. [8] In Fig. 3 the operation of the PMT can be seen in a single photoelectron state.

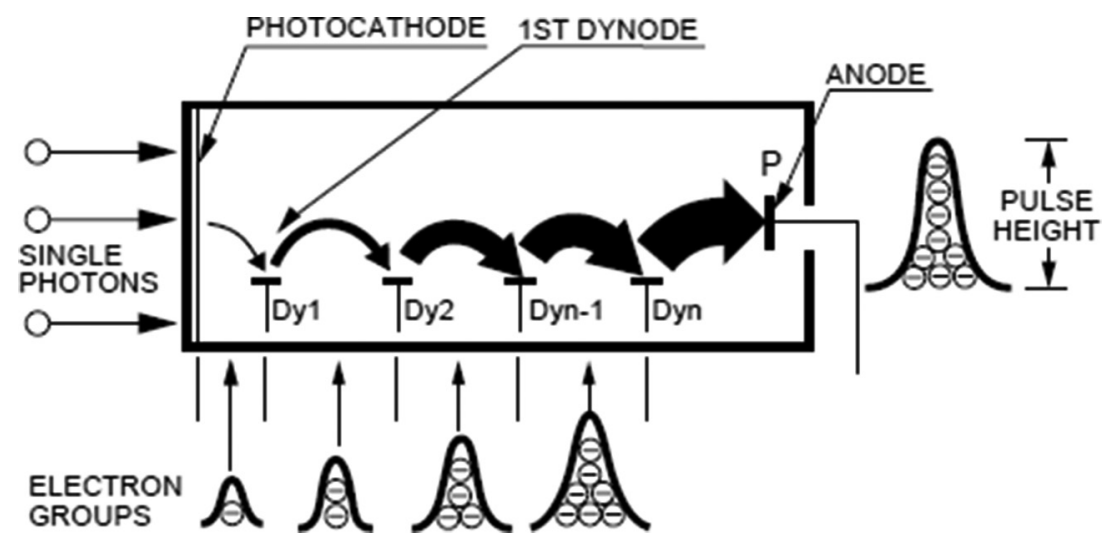

Figure 3. The operation of the PMT in a single photoelectron state. [8]

The Single Electron Response (SER) is also a very important definition that has to be discussed to understand the basics of single photon detection. Based on the situation that the maximum continuous output current (Imax) is much lower than the peak current (ISER), the PMT is operated near its full gain. This means that the PMT produces random pulses. Each of the pulses represents the detection of a single photon. The signal's pulse density at the cathode of the detector provides the measure of light intensity. The SER of the PMT is the output pulse for a single photoelectron. [11: 6]

The peak current (ISER) can be calculated from the following equation:

$$
I_{S E R}=\frac{\mathrm{G} \cdot \mathrm{e}}{T_{S E R}}
$$

where $\mathrm{T}_{\mathrm{SER}}$ is the SER pulse width (fwhm), G is the PMT gain (standard PMT has $10^{7}$ ) and e is the charge of an electron $\left(1,6 \times 10^{-19} \mathrm{C}\right)$. [11: 223] 
In Picture 3, the power controller and the Millenia Pro green laser can be seen, it is connected to the Mira 900 Ti:Sapphire laser.

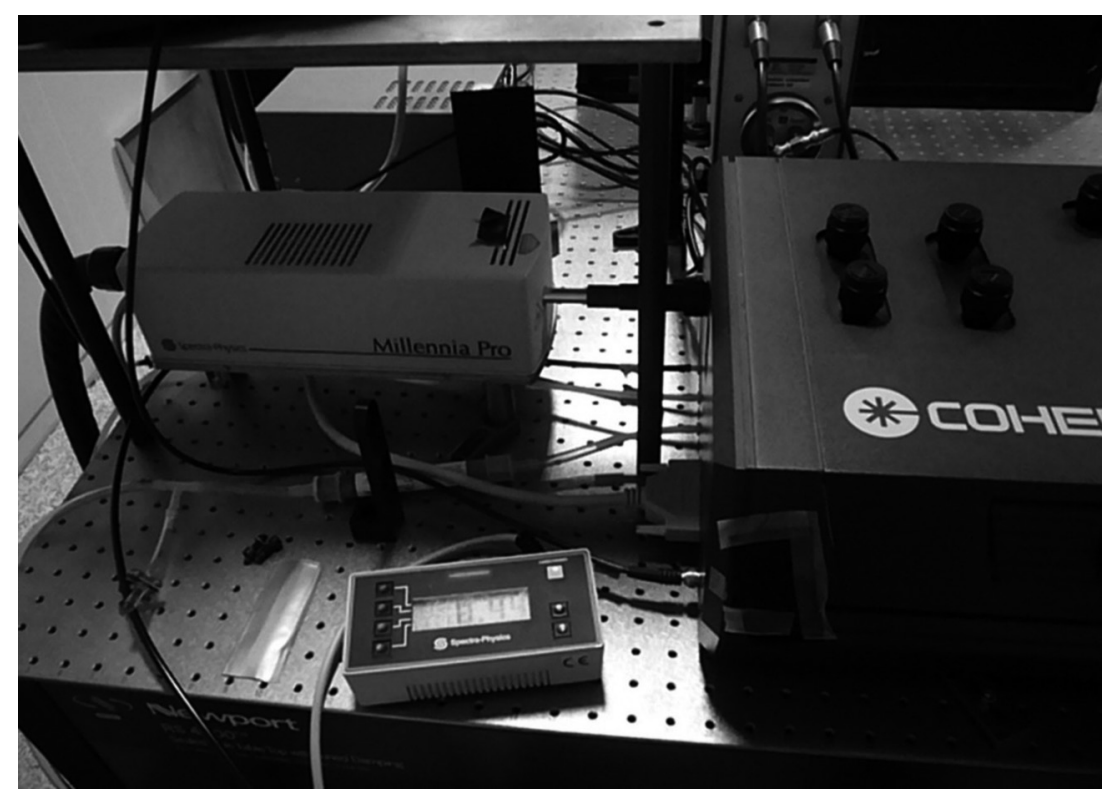

Picture 3. Power controller of the Mira 900 Ti:sa laser. (Made by author.)

\section{The SR 400 photon counting system}

In Fig. 4 it can be seen that the SR400 combines amplifiers, discriminators, gate generators and counters all together. The channels inside the counter can count even at rates up to 200 $\mathrm{MHz}$. There are two independent channels in the SR400 system. Each counting channel has its own gate generator which provides counting gates between $5 \mathrm{~ns}$ and $1 \mathrm{~s}$. The counters named A, B and T are the fast counters. All three counters operate at rates up to $200 \mathrm{MHz}$. From a number of sources the input is selected for each counter and includes the two analogue signal inputs. These are the external trigger input and the $10 \mathrm{MHz}$ crystal timebaser. Counter $\mathrm{T}$ can be present to determine the measurement period. [12] Fig. 4 shows a very similar photon counting system to that which has been used for the measurements.

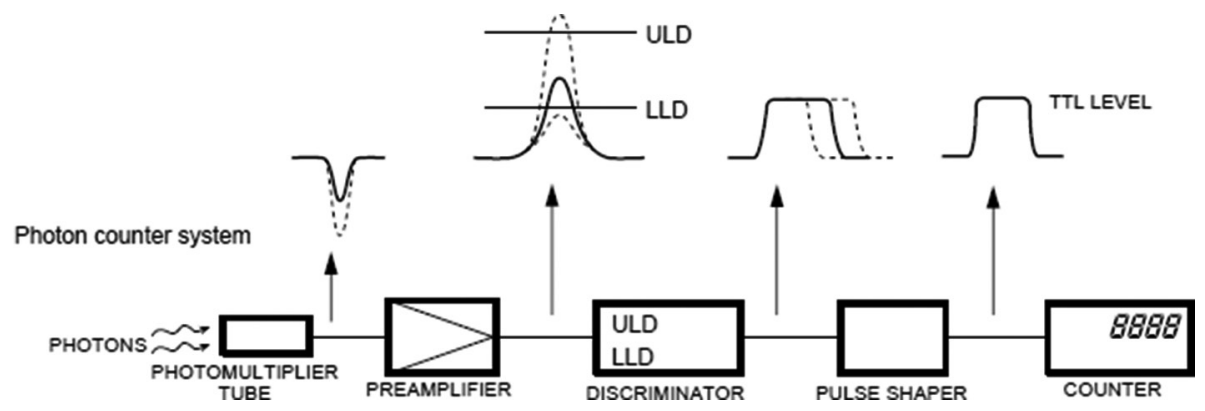

Figure 4. Typical photon counting system. [8] 
In Picture 4, the display of SR400 photon counter can be seen which currently shows 27 impacted photons at a certain state of the rotator stage with 1 sec scan time.

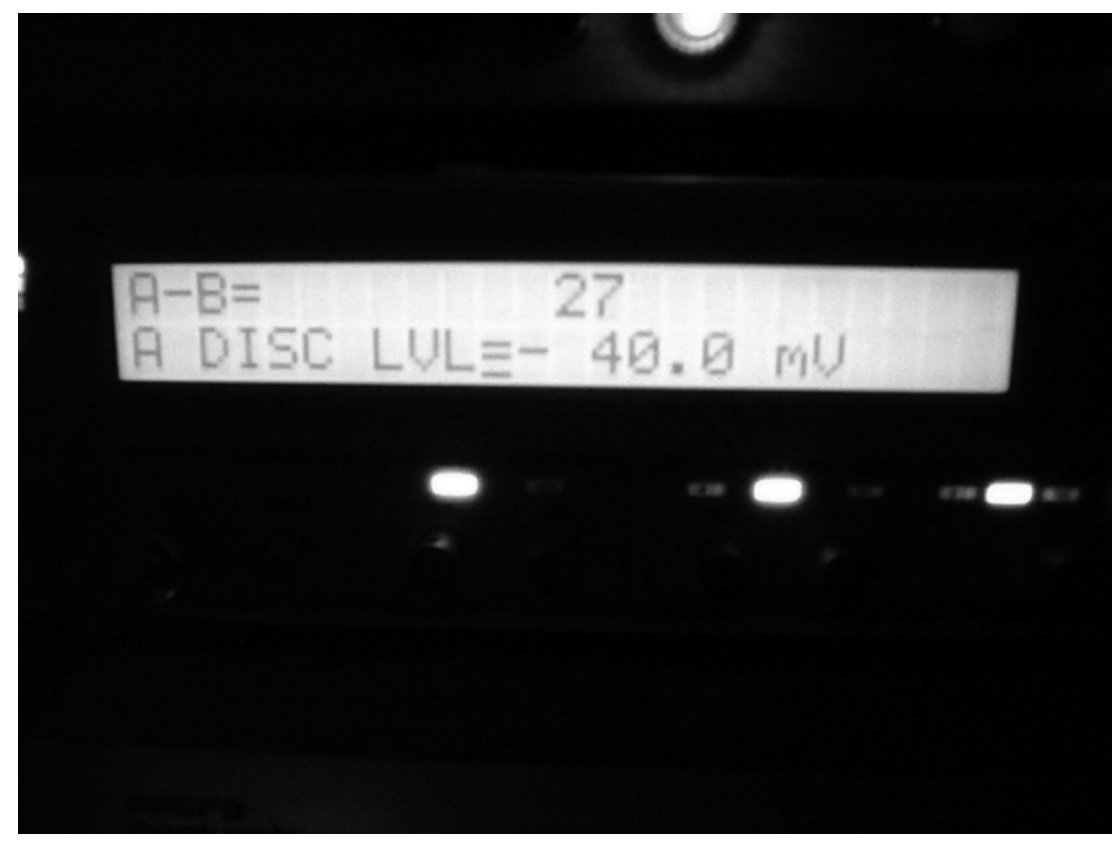

Picture 4. SR 400 photon counter currently shows 27 impacted photons at a certain degree. (Made by author.)

To extend the events via the 2 gate generators (which are independent), the A and $\mathrm{B}$ counters may also be synchronized in pulsed experiments. The gate generator provides gates from 5 ns to 1s in duration with a delay from an external trigger ranging from 25 ns to $1 \mathrm{~s}$. In a single scan, 1 to 2000 count periods can be cycled through with the SR400 photon counter and it can detect pulses as low as $2 \mathrm{mV}$. [12] It is practical to set the pulse size to $10 \mathrm{mV}$ to provide better noise immunity and to allow for some adjustment of the discriminator threshold. Between $10 \mathrm{mV}$ and $300 \mathrm{mV}$ the preamplifier should have enough gain to amplify anode pulses. The $100 \mathrm{mV}$ is probably the best target value.

\section{Analysis of SHG signals from Si (111) sample and results}

The signals SH origin can be checked by measuring the intensity at a certain position of the sample (at a fixed rotation angle) by changing the intensity of the laser with a polarizer, step by step. At the same time we have to use a certain amount of special short wave pass filters after the sample to avoid any other noises like two photon luminescence. In this case after some measurements we can fit a linear function to the values and the exponent value (B) must be 2. If it is approximately 2, then we can be sure that the signals are really Second Harmonic.

The other way to check SHG is to use a monochromator. Also the background noises have to be checked by blocking the laser beam. 
The discriminator compares the input pulses with the present reference voltage to divide them into two groups: one group is lower and the other is higher than the reference voltage. The lower pulses are eliminated by the lower level discriminator and in the same cases, the higher pulses are eliminated by the upper level discriminator. The detector system operates at negative voltage and this is the reason why the discriminator voltages should also be negative. The signals have been found to be the most optimal with the A level at $-40 \mathrm{mv}$ and the B level at $-155 \mathrm{mv}$.

For expectation we have to use the theoretical model of (111) structures. For each polarization configuration we have to use a certain function to find what we expect. The following equations have been derived from the theoretical model of (111) structures [13]:

At SS configuration: $\mathrm{I}^{2 \omega}(\varphi)=(\mathrm{A} \times \sin 3[\varphi-\varphi 0])^{2}$

At SP configuration: $\mathrm{I}^{2 \omega}(\varphi)=(\mathrm{A}+\mathrm{B} \times \sin 3[\varphi-\varphi 0])^{2}$

At PP configuration: $\mathrm{I}^{2 \omega}(\varphi)=(\mathrm{A}+\mathrm{B} \times \sin 3[\varphi-\varphi 0])^{2}$

At PS configuration: $\mathrm{I}^{2 \omega}(\varphi)=(\mathrm{A} \times \sin 3[\varphi-\varphi 0])^{2}$

where $\mathrm{I}^{2 \omega}(\varphi)$ is the second harmonic intensity and $\varphi$ is the rotation angle.

Table 1. This table shows the components of the surface nonlinear polarization of centrosymmetric crystals of classes m3m and 432. [13]

\begin{tabular}{l|c|c}
\hline \multicolumn{2}{|c}{$P_{1}^{s}(2 \omega)(111)$} \\
\hline \multicolumn{1}{|c|}{ Isotropic } & \multicolumn{1}{c}{ Anisotropic } \\
\hline $\begin{array}{l}E^{2}\left[-\chi_{x^{\prime} x^{\prime} z^{\prime}}^{S} \sin 2 \theta_{2} \cos ^{2} \psi \cos \varphi_{2}+\left\{\cos ^{2} \psi\right.\right. \\
\left(\chi_{z^{\prime} z^{\prime} z^{\prime}}^{S} \sin ^{2} \vartheta_{2}+\chi_{z^{\prime} x^{\prime} x^{\prime}}^{S} \cos ^{2} \vartheta_{2}\right. \\
\left.\left.+\chi_{z^{\prime} x^{\prime} x^{\prime}}^{S} \sin ^{2} \psi\right\} \sin \varphi_{2}\right] \\
E^{2}\left(-2 \chi_{x^{\prime} x^{\prime} z^{\prime}}^{S} \sin \vartheta_{2} \sin \psi \cos \psi\right)\end{array}$ & $\begin{array}{c}E^{2} \chi_{x^{\prime} x^{\prime} x^{\prime}}^{S}\left\{\cos ^{2} \theta_{2} \cos ^{2} \psi \cos 3 \varphi\right. \\
-\sin ^{2} \psi \cos 3 \varphi-2 \cos \theta_{2} \cos \psi \sin \psi \\
\times \sin 3 \varphi\} \cos \varphi_{2}\end{array}$ \\
& $\begin{array}{l}E^{2}\left(-\chi_{x^{\prime} x^{\prime} x^{\prime}}^{S}\right)\left\{\cos ^{2} \theta_{2} \cos ^{2} \psi \sin 3 \varphi\right. \\
-\sin ^{2} \psi \sin 3 \varphi+2 \cos \vartheta_{2} \cos \psi \sin \psi \\
\times \cos 3 \varphi\}\end{array}$
\end{tabular}

We can determine the $\mathrm{P}(2 \omega)$ for the volume and surface layers of the (111) planes for arbitrary polarization of the pump radiation. $\mathrm{X}_{\mathrm{i}^{\prime} \mathrm{j}^{\prime} \mathrm{k}^{\prime}}$ are the components of the surface susceptibility tensor in the x'y'z' coordinate system; 1 is the polarization index of the SH radiation; $\mathrm{E}$ is the amplitude of the pump field in the nonlinear medium; $\varphi_{2}, \mathrm{v}_{2}$ are the angles between the surface normal and the wave vectors of this SH and pump respectively; $\mathrm{k}$ is the modulus of the wave vector of the pump radiation in the nonlinear medium. The polarization angle is $\psi$, the rotation angle is $\varphi$. [13]

The incident angle in our case is 45 degrees. 
For s-polarized pump radiation (the angle $\psi=\pi / 2$ ) the isotropic s-component of the nonlinear polarization vector equals zero. This implies that when the nonlinear interaction takes place in the s-s geometry, there is no isotropic SH for the crystals we are studying (the classes $\mathrm{m} 3 \mathrm{~m}$ and 432). This applies to the surface dipole and volume quadrupole $\mathrm{SH}$ to the same degree. [13]

The methods at certain polarization configurations are the following:

$\mathrm{X}$ is the first polarization position and the second can be $\mathrm{P}$ or $\mathrm{S}$

$1, \mathrm{X}=\mathrm{P} \rightarrow \psi=0$

$2, X=S \rightarrow \psi=\pi / 2$

We can use these values for the table contents (see Table 1).

The following SHG measurements have been made with 1 sec scan time and 1 degrees step size using $800 \mathrm{~nm}$ laser beam. The power is $400 \mathrm{~mW}$. It has been realized that the signals are also strongly dependent on the stability of the Mira 900 Ti:Sapphire laser.

Due to this fact the next step was to optimize the alignment of the laser (find the maximum power, where the laser is still stable and pulsed). Usually the maximum output power was $400-420 \mathrm{~mW}$ at $800 \mathrm{~nm}$ wavelength.

Fig. 5 shows the SHG curves at SS polarization configuration. The highest number of the impacted photons was around 2550. The number of impacting photons also depends on the certain position (in degrees) of the rotator stage. The size and the number of the certain peaks depend on the polarization configuration. With this configuration six, very similar peaks can be observed.

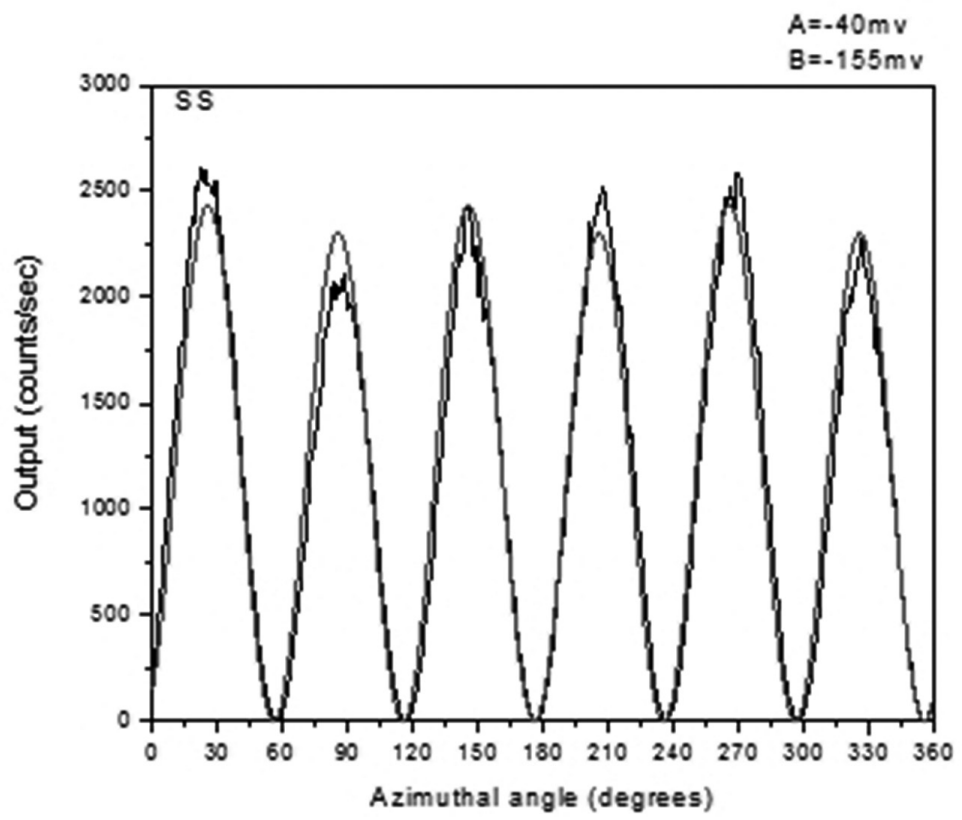

Figure 5. Second Harmonic intensity at SS polarization configuration in the function of rotation angle with the expectation curves using equation 7. (Made by author.) 
The curve in Fig. 6 was made with SP polarization configuration, where three main peaks could be observed. At 178 degree position the highest number of impacted photons was detected.

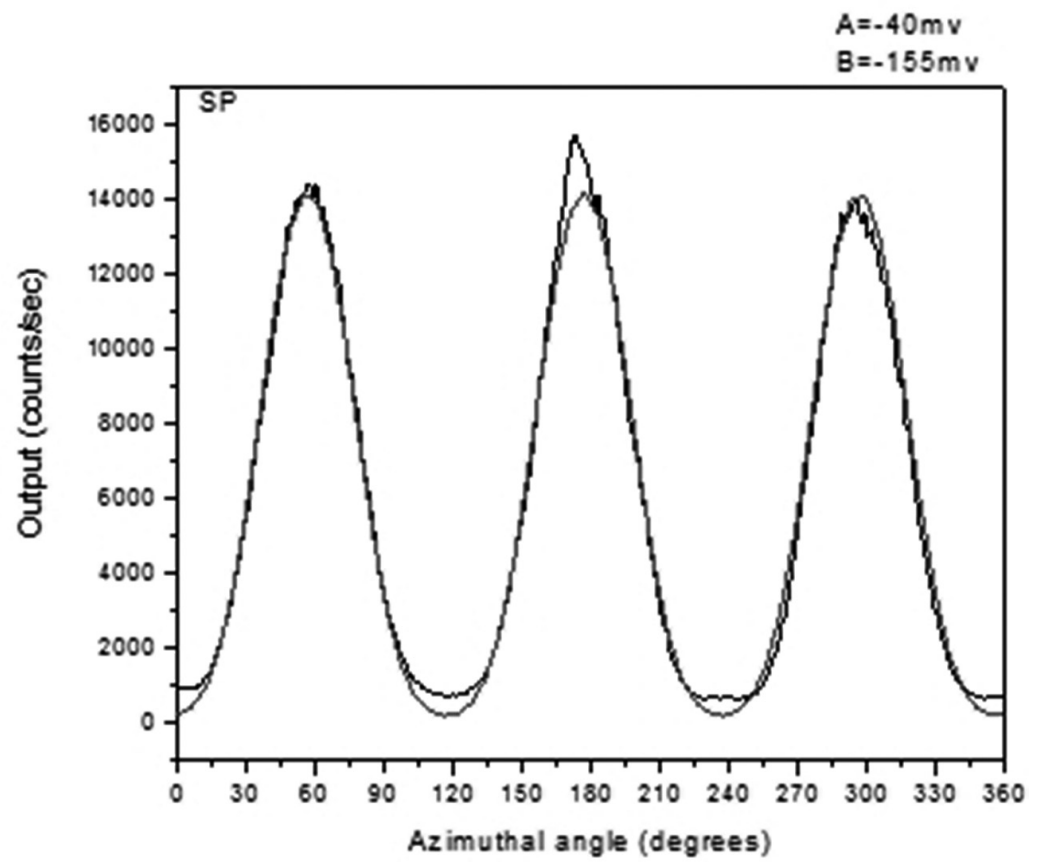

Figure 6. Second Harmonic intensity at SP polarization configuration in the function of rotation angle with the expectation curves using equation 8. (Made by author.)

In Fig. 7 the curve at PP polarization configuration can be seen. There are two small peaks at 120 and 240 degree position and three high peaks at 60, 180 and 300 degree position. The highest number of detected pulses was around 3200. 


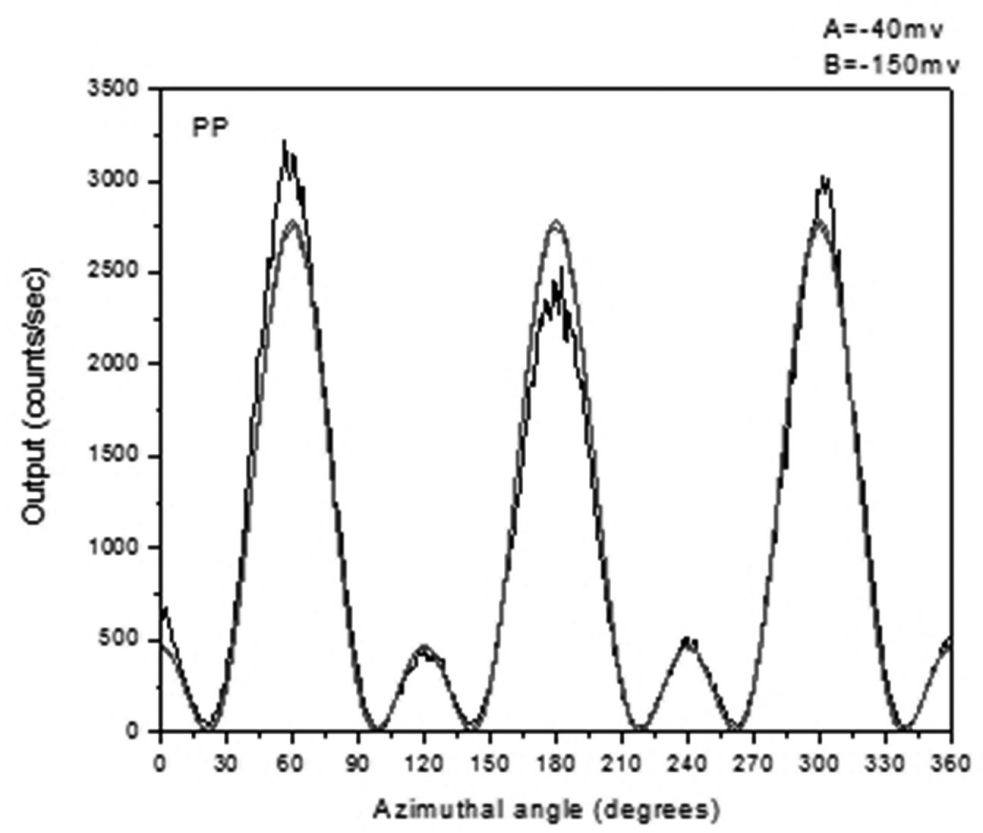

Figure 7. Second Harmonic intensity at PP polarization configuration in the function of rotation angle with the expectation curves using equation 9. (Made by author.)

Finally the SHG intensity at PS polarization configuration can be seen in Fig. 8. The maximum value of the detected pulses rates up to 1600 only at 150 degrees position.

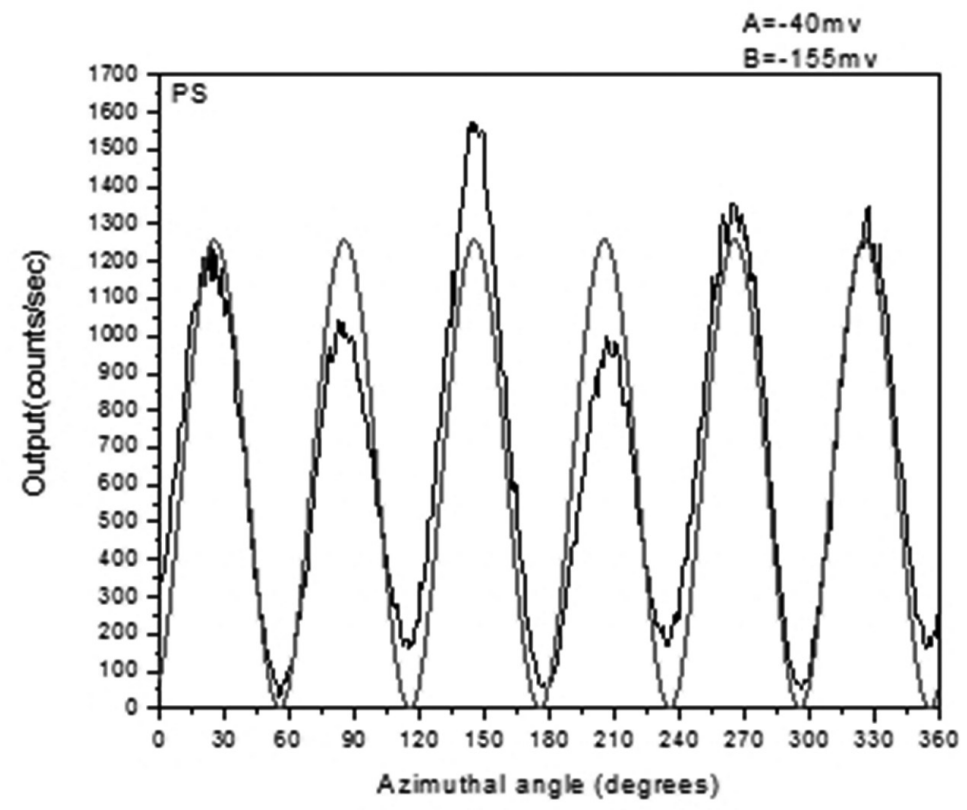

Figure 8. Second Harmonic intensity at PS polarization configuration in the function of rotation angle with the expectation curves using equation 10. (Made by author.) 


\section{Photon counting in a different way}

A very promising task is to build such a setup that can be used for communication between robotic vehicles. In order to decrease the size of the whole setup, it is extremely important that there must be a different laser light source with another stable, but much lighter power supply. For this experiment the use of a single photon avalanche diode (SPAD) is one of the most practical ways to count the impacting photons. This has a higher QE compared to PMTs. Depending on the manufacturer, sometimes the QE of an SPAD can be even $80 \%$. SPADs have some disadvantages, such as the light having to be focused into it precisely and it must be kept at a cold temperature. The cooling method can be achieved with small peltier batteries, which have been tried already with InGaAs avalanche photodiodes. [14] The communication between robotic vehicles (mainly in military applications) should be secret. To achieve this, an already published experimental setup could be used in the opposite way. [15] As earlier published, the development of the antibunching photon contains an optical delay system to detect only one photon. This experiment works with an Optical Parametric Oscillator (OPO) and with a nonlinear crystal that divides the impacting photon to form new photons with half the energy, and therefore half the frequency and double the wavelength of the initial photons. In this experiment the original wavelength of the initial photons was $400 \mathrm{~nm}$.

\section{Conclusions}

Experiments with SHG have been done with the four main polarization configurations. It has been proven that photon counting with special devices is possible from one up to 300 million photons. With SP configuration the highest number of the initial photons was detected. The counter was able to count up to 16000 initial pulses. With the opposite (PS) configuration the lowest number of the initial photons have been detected. With this the count value was up to 1600. So at SP configuration we can expect 10 times more impacting photons on certain degrees than at PS configuration. Checking these results we can declare that the expectation curves match well to the individual polarization configurations except the PS configuration, but here the original signal was not as good as expected.

What has been shown here is a certain case of photon counting with the experiment of SHG. To apply the photon counting methods on robotic vehicles for photonic communications (such as the use of the antibunching photon), the other way around can be also an acceptable method with a different counter and with an SPAD. In the case of the antibunching photon with the use of an OPO the frequency must be half of the original wavelength to form new photons with half the energy, and therefore half the frequency and double the wavelength of the initial photons. 


\section{References}

[1] URSIN, R. et al.: Free-Space distribution of entanglement and single photons over $144 \mathrm{~km}$. Ithaca: Cornell University, 2007. www.arxiv.org/pdf/quant-ph/0607182 (downloaded: 0810 2014) DOI: https://doi.org/10.1038/nphys629

[2] FEYNMAN, R. P., LEIGHTON, R. B., SANDS, M.: Feynman Lectures on Physics. Vol. 2, Boston: Addison-Wesley, 1964.

[3] DOLUWEERA, S.: The Role of Electric Polarization in Nonlinear optics.

Cincinnati: University of Cincinnati, 2001. www.phys.lsu.edu/ jarrell/COURSES/

ELECTRODYNAMICS/Student_Projects/sumith/sumith-talk.pdf. (downloaded: 2511 2014)

[4] SHEN Y. R.: Wave Mixing Spectroscopy for Surface Studies. Solid State Communications, 102221 (1997). DOI: https://doi.org/10.1016/S0038-1098(96)00726-0

[5] PASCHOTTA R.: Encyclopedia of Laser Physics and Technology-Frequency Doubling. New York: Wiley-VCH, 2008.

[6] MCMAHON, M. D. et al.: Second harmonic generation from resonantly excited arrays of gold nanoparticles. Applied Physics B, 87 (2007), 259-265. DOI: https://doi.org/10.1007/ s00340-006-2569-3

[7] HUBERT C. et.al.: Role of surface plasmon in second harmonic generation from gold nanorods. Applied physics letters, 90181105 (2007). DOI: https:// doi.org/10.1063/1.2734503

[8] HAMAMATSU: Photon counting using photomultiplier tubes. Toyooka: Hamamatsu Photonics K. K., Electron Tube Center, 1998. http://123.physics.ucdavis.edu/shot_files/ photoncounting.pdf (downloaded: 0810 2014)

[9] BECKER, W., BERGMANN A.: Detectors for High-Speed Photon Counting. Berlin: Becker \& Hickl GmbH, s.d. www.becker-hickl.de/pdf/spcdetect1.pdf (downloaded: 0411 2014)

[10] SRS: Signal Recovery with PMTs. Sunnyvale: Stanford Research Systems, Inc., s.d. www. thinksrs.com (downloaded: 0411 2014)

[11] BECKER, W.: Advanced time correlated single photon counting techniques (springer series in chemical physics). New York: Springer, 2005.

[12] SRS: SR400 - Gated Photon Counter (2-channel). www.thinksrs.com/downloads/PDFs/ Catalog/SR400c.pdf (downloaded: 0810 2014)

[13] AKTSIPETROV, O. A., BARANOVA, I. M., IL'INSKII, Y. A.: Surface contribution to the generation of reflected second harmonic light for centrosymmetric semiconductors. $Z h$. Eksp. Teor. Fiz., 91 (1986), 287-297. www.jetp.ac.ru/cgi-bin/dn/e_064_01_0167.pdf (downloaded: 0810 2014)

[14] ZHOU P. Y. et al.: Single-photon property characterization of $1.3 \mu \mathrm{m}$ emissions from InAs/ GaAs quantum dots using silicon avalanche photodiodes. www.ncbi.nlm.nih.gov/pmc/ articles/PMC3887382/\#!po=2.08333 (downloaded: 2810 2014) DOI: https:// doi.org/10.1038/srep03633

[15] KURILLA B.: A lézerek alapjai, lézerfizikai eszközök és alkalmazásaik robottechnikai eszközökön. Hadmérnök, IX 1 (2014), 217-229. http://hadmernok.hu/141_21_kurillab.pdf (downloaded: 0411 2014) 